\title{
Einführung in die Diagnostik humanpathogener Pilze - Teil 3: Fakultativ-pathogene Sprosspilze (Hefen)
}

\author{
Introduction to the Diagnosis of Human Pathogenic Fungi - \\ Part 3: Opportunistic Pathogenic Yeasts
}

Autoren

Institut
A.-M. Ksoll, B. Sorhage

Abteilung Dermatologie, Venerologie und Allergologie, Bundeswehrzentralkrankenhaus Koblenz
Bibliografie

DOI http://dx.doi.org/

10.1055/s-0031-1291479

Akt Dermatol 2011; 37: 441-450

(c) Georg Thieme Verlag KG

Stuttgart · New York

ISSN 0340-2541

Korrespondenzadresse

Anne-Marie Ksoll

Biomed. Fachanalytikerin und Fachlehrerin (LGW-Univ.)

\section{Dr. med. Bernhard Sorhage}

Abteilung Dermatologie, Venerologie und Allergologie Bundeswehrzentralkrankenhaus Koblenz

Akademisches Lehrkrankenhaus der Johannes Gutenberg Universität Mainz Rübernacher Straße 170 56072 Koblenz

\section{Zusammenfassung \\ $\nabla$}

Die Hefepilze sind weltweit verbreitet und kommen häufig in Nahrungsmitteln, vor allem in Milchprodukten, aber auch in der Luft, im Wasser und im Erdboden vor. Hefepilze, die keine sexuellen Fruchtformen bilden und sich nur ungeschlechtlich durch Sprossung vermehren, werden im Gegensatz zu den perfekten, askosporenbildenden Hefen, den sogenannten Askomyzeten, in ein künstliches System der „Fungi imperfecti“ eingeordnet und als Deuteromyzeten bezeichnet. In der Humanmedizin sind vor allem die imperfekten Hefen bzw. Sprosspilze der Gattung Candi-

Zur Untersuchung gelangen in erster Linie Schleimhautabstriche, ferner Urin und Stuhlproben, gelegentlich auch Blut und Liquor. Bei Verdacht auf Hefepilzinfektionen des äußeren Integuments werden Hautschuppen, Nagelspäne und Haarstümpfe sowie Hautabstriche von der Perianalgegend und der Glans penis untersucht.

Die Hefepilze werden primär zumeist auf einfachen, kostengünstigen Festmedien ohne Actidion-(Cycloheximid)-Zusatz, z. B. Sabouraud-Glukose- $(2 \%)$ oder Kimmig-Agar angezüchtet und anschließend auf Reisagar ( $\bullet$ Abb.1) subkultiviert.

Die Hefekolonien sind von weich-pastöser oder schleimiger Konsistenz und können weißlich-cremefarben bis gelblich-braun oder rosa bis rötlich pigmentiert sein.

Die in den Primärkulturen gewachsenen, verschiedenen Hefen sehen einander so ähnlich, dass eine Artdiagnose überhaupt nicht gestellt werden kann. Selbst eine Einordnung nach der Gattung ist nur in wenigen Fällen aus Oberflächenbild, Farbe und Rand einer Kolonie möglich [1].

Die Identifizierung der Hefepilze erfolgt durch morphologische Beurteilung der auf Reisagar gebildeten charakteristischen Mikrostrukturen so- da, Cryptococcus, Malassezia (Pityrosporum) sowie mit Einschränkung Rhodotorula und Sporobolomyces von Bedeutung. Die umfangreichste Gattung ist die von Candida mit etwa 200 Arten. Nur wenige dieser imperfekten Hefen sind fakultative Parasiten und besitzen die Fähigkeit bei oder über $37^{\circ} \mathrm{C}$ zu wachsen. Diese opportunistisch-pathogenen Erreger können bei Bestehen disponierender Faktoren Hefemykosen (Levurosen) beim Menschen hervorrufen und haben vor allem seit der Einführung der antibakteriellen Antibiotika, Zytostatika, Immunsuppressiva und anderer iatrogener Maßnahmen eine zunehmende Bedeutung erlangt.

wie durch Prüfung biochemischer Eigenschaften (Fermentations- und Assimilationsleistungen). Für die biochemische Differenzierung der Hefepilze sind Reinkulturen erforderlich.

Die Assimilations- und Fermentationsleistungen können mithilfe verschiedener diagnostischer Testkits bestimmt werden. Biologische Abweichungen sind jedoch möglich. Zur Absicherung der Untersuchungsergebnisse sind infolgedessen noch zusätzlich die mikromorphologischen Kriterien auf Reisagar zu berücksichtigen.

Drei der medizinisch wichtigsten Candida-Arten, Candida (C.) albicans, C. krusei und C. tropicalis können auch mithilfe der Selektivnährböden Chromagar-Candida oder ChomID-Candida identifiziert werden, die ein farblich unterschiedliches Kolonienwachstum dieser Pilze ermöglichen.

\section{Gattung Candida \\ $\nabla$}

In dieser Gattung sind etwa 200 Arten bekannt. Die Gattung Candida umfasst morphologisch und physiologisch ähnlich erscheinende Pilze, die aber in vielen Fällen in keinerlei Verwandtschaftsverhältnis untereinander stehen [2]. 
Diese weit verbreiteten Sprosspilze kommen häufig an Standorten mit reichem Zuckerangebot, wie z. B. auf Blüten und Früchten, aber auch in Nahrungsmitteln, vor allem in Milch und Milchprodukten vor.

Man unterscheidet 17 fakultativ-pathogene Arten. Candida albicans stellt die medizinisch weitaus wichtigste Candida-Spezies dar. Andere, seltener für den Menschen als Krankheitserreger relevante Candida-Arten sind vor allem C. glabrata, C. guilliermondii, C. krusei, C. parapsilosis und C. tropicalis.

Voraussetzung für das Auftreten einer Candidose ist das Vorliegen gewisser disponierender Faktoren, die den opportunistischpathogenen Charakter des Erregers in einen parasitären transformieren [3].

Die Entstehung einer disseminierten Candida-Infektion wird vor allem durch Malignome, bauchchirurgische Eingriffe, Diabetes mellitus, HIV-Infektionen, lang anhaltende Leukopenien, Darmerkrankungen, i.v. Drogenabhängigkeit sowie iatrogene Maßnahmen begünstigt.

Candida-Arten bilden weißlich-cremefarbene, halbkugelig gewölbte Kolonien mit glatter, manchmal leicht faltiger oder gekräuselter Oberfläche. Der Kolonienrand kann gelegentlich von strahlenförmig auslaufenden Pseudomyzelhyphen umgeben sein.

\section{Candida albicans}

Synonym: Syringospora robinii, Syringospora albicans, Monilia albicans und nahezu 100 weitere Synonyma

Candida albicans ( $\mathbf{A b b . 2 - 4}$ ) ist ein Endosaprophyt des MagenDarm-Traktes und der Vagina. Dieser Sprosspilz kann sich je nach lokaler bzw. allgemeiner Abwehrlage des bereits vorgeschädigten Wirtsorganismus in unphysiologischer Weise vermehren und zu einem lebensbedrohenden Problemkeim werden. Die Infektionen manifestieren sich auf den Schleimhäuten, in Form grauweißer, pilzrasenartiger Beläge (sogenannter Soor), der Haut sowie ihren Anhangsgebilden und nach Disseminierung der Erreger auf hämatogenem Wege an den inneren Organen.

Candida albicans ist aufgrund ihres biochemischen Verhaltens sowie der Chlamydosporen- und Keimschlauch- bzw. Pseudomyzelbildung in Seren nach 2- bis 4-stündiger Inkubation bei $37^{\circ} \mathrm{C}$ relativ leicht zu identifizieren $(\bullet$ Abb.5).

Mikromorphologie: Candida albicans bildet meist reichlich Pseudomyzel mit runden bis ovalen, traubenförmig im Bereich der Pseudomyzelgrenzen angeordneten Blastosporen ( $\bullet$ Abb.6) und überwiegend terminal inserierten Chlamydosporen ( $\bullet$ Abb. 7).

Tab. 1 Biochemisches Verhalten von Candida albicans.

\begin{tabular}{|c|c|c|c|}
\hline Fermentation & & & Assimilation \\
\hline Glukose + & Laktose & Glukose + & Laktose - \\
\hline Galaktose V & Raffinose & Galaktose + & Raffinose - \\
\hline Saccharose V & & Saccharose + & Trechalose + \\
\hline Maltose + & & Maltose + & Nitrat - \\
\hline
\end{tabular}

\section{Candida glabrata}

Bekanntestes Synonym: Torulopsis glabrata

Candida glabrata ( Abb.8) wird in Schleimhautabstrichen des Urogenitaltrakts, vor allem der Vagina relativ häufig nachgewiesen. Im Rahmen der mykologischen Harndiagnostik ist Candida glabrata nach Candida albicans der wichtigste Sprosspilz [4]. Befallen werden von dieser Candida-Spezies auch der Intestinalund Respirationstrakt.
Mikromorphologie: Die rundlichen bis geringovalen Blastosporen sind relativ klein ( $\bullet$ Abb.9). Pseudomyzelien und Myzelien werden nicht gebildet. In sehr seltenen Fällen wurde ein rudimentäres Pseudomyzel beschrieben.

Tab. 2 Biochemisches Verhalten von Candida glabrata.

\begin{tabular}{llll} 
Fermentation & & Assimilation \\
\hline Glukose + & Laktose - & Glukose + & Laktose - \\
\hline Galaktose - & Raffinose - & Galaktose - & Raffinose - \\
Saccharose - & & Saccharose - & Trechalose V \\
Maltose - & & Maltose - & Nitrat -
\end{tabular}

\section{Candida guilliermondii}

\section{Perfektes Stadium: Pichia guilliermondii}

Candida guilliermondii ( $\bullet$ Abb. 10) ist ein auf der menschlichen Haut und Schleimhaut gelegentlich vorkommender Saprophyt. Dieser Sprosspilz kann sich unter bestimmten Bedingungen auch in unphysiologischer Weise vermehren und zu klinischen Erscheinungen führen. Bevorzugt befallen werden von dieser Candida-Spezies die Haut und ihre Anhangsorgane.

Mikromorphologie: Die sehr kleinen Blastosporen sind oval bis kurz-zylindrisch. In älteren Kulturen liegen sie so zahlreich und dicht beieinander, dass sie den Eindruck eines ballförmigen Gebildes vermitteln [5]. Pseudomyzel wird zumeist nur spärlich ge-

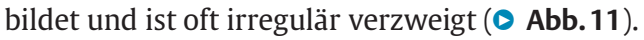

Tab.3 Biochemisches Verhalten von Candida guilliermondii.

\begin{tabular}{|c|c|c|c|}
\hline \multicolumn{3}{|l|}{ Fermentation } & \multirow{2}{*}{$\begin{array}{l}\text { Assimilation } \\
\text { Laktose V }\end{array}$} \\
\hline Glukose + & Laktose - & Glukose + & \\
\hline Galaktose V & Raffinose + & Galaktose + & Raffinose + \\
\hline Saccharose + & & Saccharose + & Trechalose + \\
\hline Maltose - & & Maltose + & Nitrat - \\
\hline
\end{tabular}

\section{Candida krusei}

Perfektes Stadium: Pichia fermentans

Synonym: Pichia krusei, Pichia kudriavzevii

Candida krusei ( $\bullet$ Abb.12 und $\bullet$ Abb.13) kommt u.a. in Nahrungsmitteln, insbesondere in Milchprodukten, aber auch im Magen-Darm-Trakt von Menschen und Tieren vor und wird gelegentlich als Erreger vaginaler Candida-Mykosen, vor allem bei deren chronischen Verlaufsformen nachgewiesen.

Mikromorphologie: Candida krusei bildet relativ große, ovale bis längliche Blastosporen, die sich oft wirtelförmig um das langgestreckte, nur wenig differenzierte Pseudomyzel gruppieren. Die Pseudomyzelabschnitte erreichen eine Länge von $18-30 \mu \mathrm{m}(\bullet$ Abb.14).

Tab. 4 Biochemisches Verhalten von Candida krusei.

\begin{tabular}{|c|c|c|c|}
\hline \multicolumn{3}{|l|}{ Fermentation } & \multirow{2}{*}{$\begin{array}{l}\text { Assimilation } \\
\text { Laktose - }\end{array}$} \\
\hline Glukose + & Laktose - & Glukose + & \\
\hline Galaktose - & Raffinose - & Galaktose - & Raffinose - \\
\hline Saccharose - & & Saccharose - & Trechalose - \\
\hline Maltose - & & Maltose - & Nitrat - \\
\hline
\end{tabular}




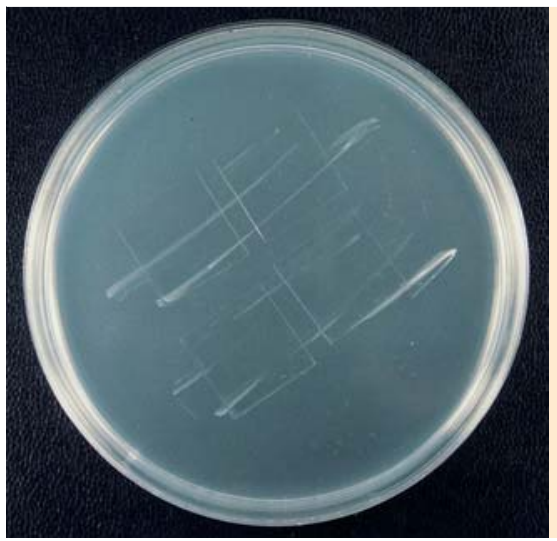

Abb.1 Reisagarkultur zur morphologischen Beurteilung der charakteristischen Mikrostrukturen der Sprosspilze.
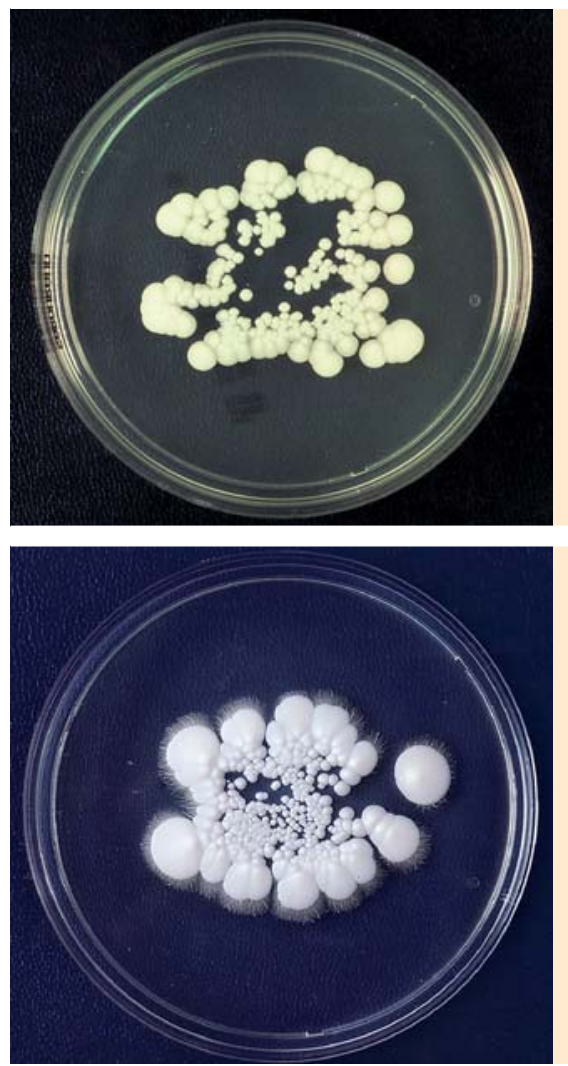

Abb. 212 Tage alte Reinkultur von Candida albicans auf SabouraudClukose-Agar (2\%) (bei $25^{\circ} \mathrm{C}$ ). Reinkultur von Candida albicans mit randständigen Pseudomyzelausläufern auf SabouraudGlukose-Agar (2\%) (bei $25^{\circ} \mathrm{C}$ ).

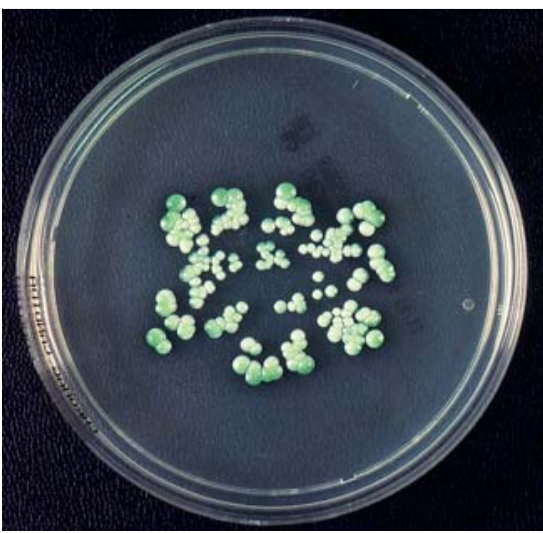

Abb.4 Candida albicans auf ChromagarCandida (nach 7 Tagen Bebrütung bei $37^{\circ} \mathrm{C}$ ).
Abb. 318 Tage alte

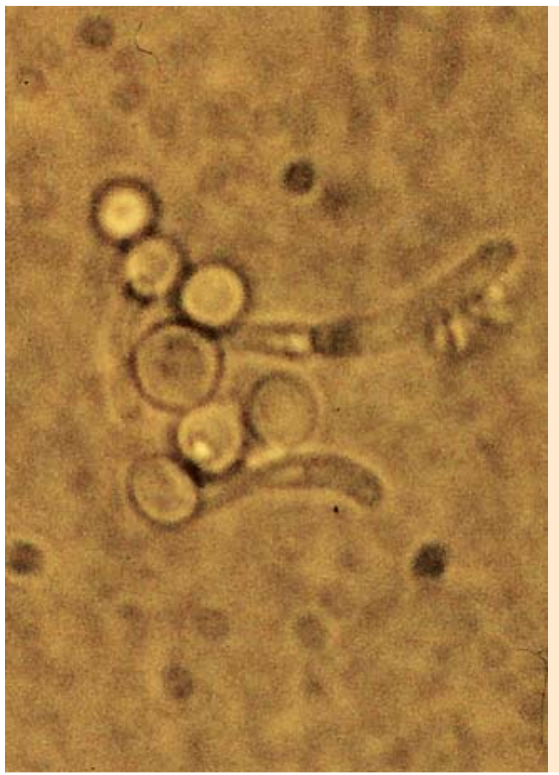

Abb.5 Keimschlauchbildung von Candida albicans in Seren nach 2-4-stündiger Inkubation bei $37^{\circ} \mathrm{C}$.

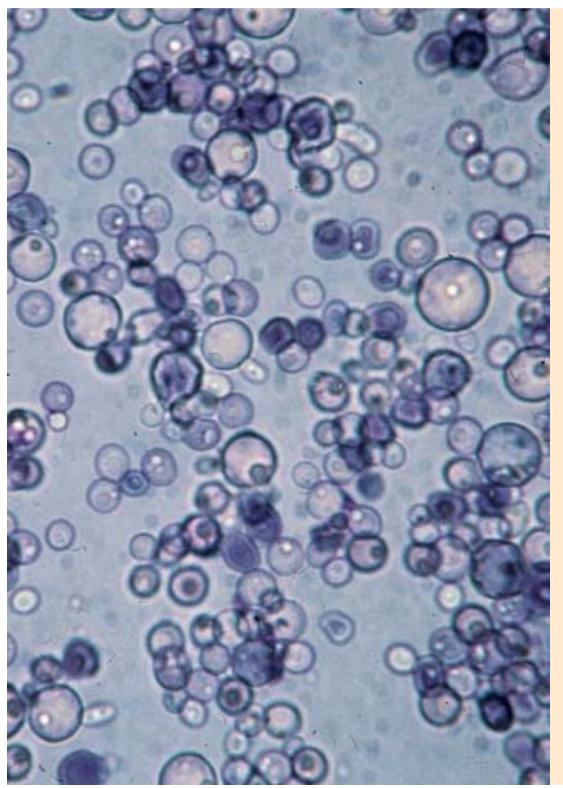
im Methylenblau-Prä-
Abb. 6 Blastosporen von Candida albicans parat (stark vergrößert).

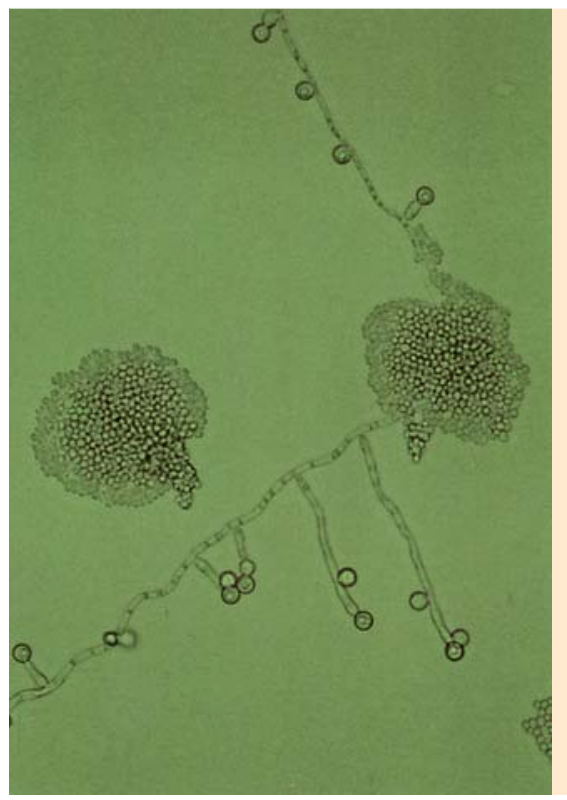




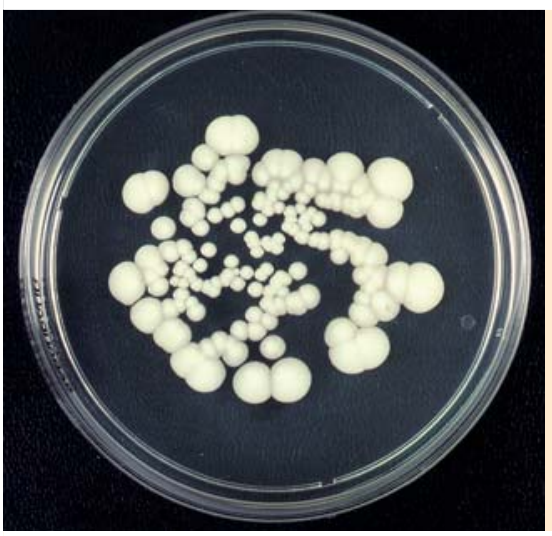

Abb. 815 Tage alte

Reinkultur von Candida glabrata auf Sabouraud-

(bei $25^{\circ} \mathrm{C}$ ).
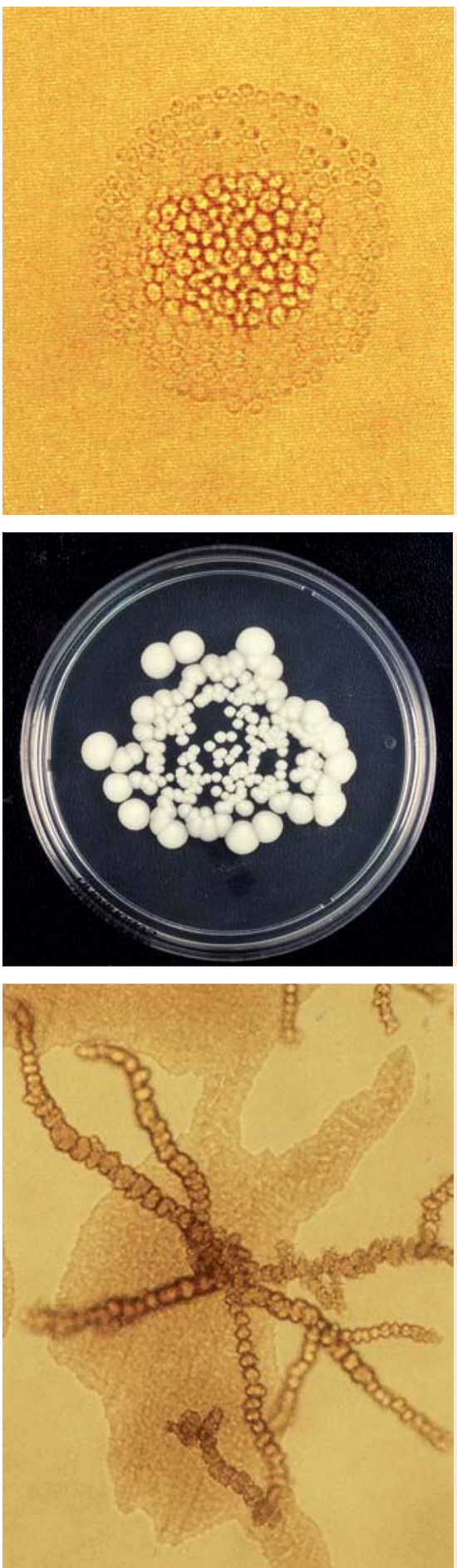

Abb.9 Mikrostrukturen von Candida glabrata auf Reisagar (stark vergrößert).

Abb.10 14 Tage alte Reinkultur von Candida guilliermondii auf Sabouraud-GlukoseAgar (2\%) (bei $25^{\circ} \mathrm{C}$ ).

Abb.11 Mikrostrukturen von Candida guilliermondii auf ReisGlukose-Agar (2\%)

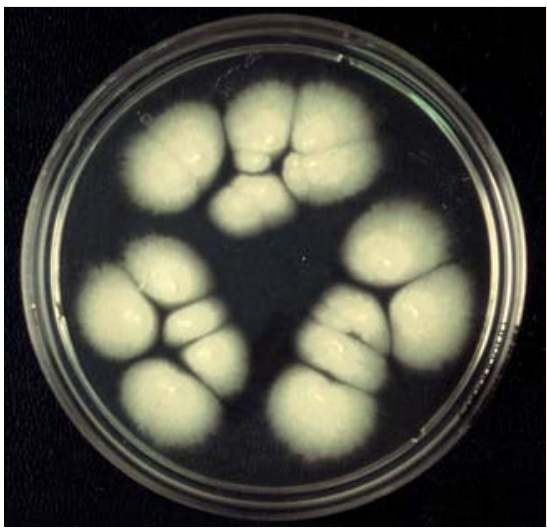

Abb.12 13 Tage alte Reinkultur von Candida krusei auf SabouraudGlukose-Agar (2\%) (bei $25^{\circ} \mathrm{C}$ ). agar (stark vergrößert).

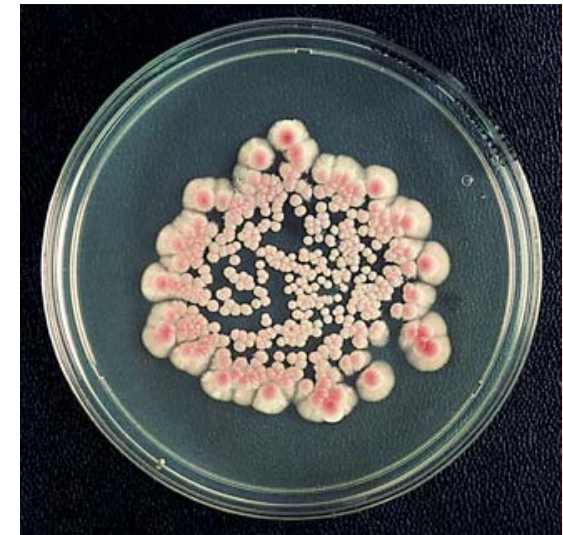

Abb.13 Candida krusei auf ChromagarCandida (nach 7 Tagen Bebrütung bei $37^{\circ} \mathrm{C}$ ).

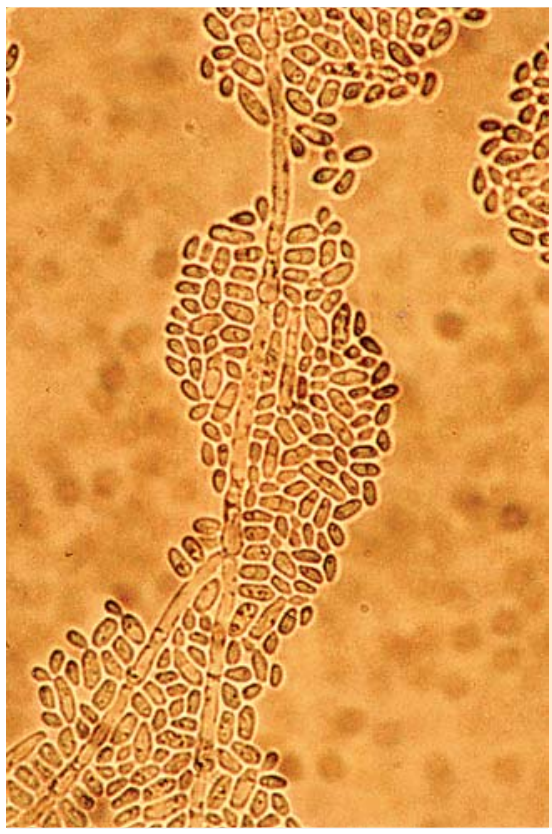

Abb. 14 Mikrostruk-

turen von Candida krusei auf Reisagar.

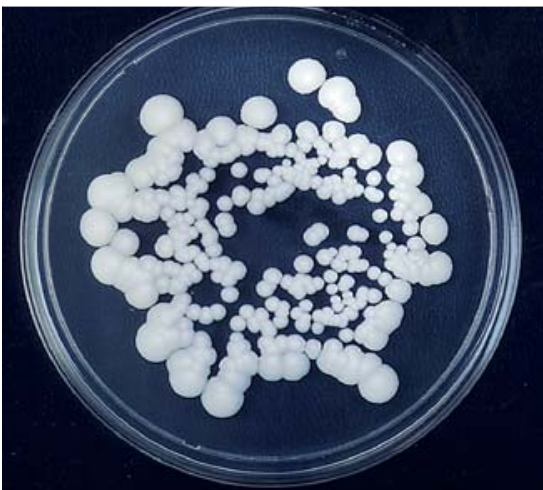

Abb.15 16 Tage alte Reinkultur von Candida parapsilosis auf Sabouraud-Glukose-Agar (2\%) (bei $25^{\circ} \mathrm{C}$ ). 


\section{Candida parapsilosis}

Candida parapsilosis ( $\bullet$ Abb.15) wird gelegentlich aus dermatologischem Untersuchungsmaterial bei Onychomykosen mit Paronychie, vor allem der Hände isoliert. Dieser Sprosspilz konnte auch mehrfach als Erreger von pilzbedingten Sepsis- und Endokarditisfällen identifiziert werden.

Mikromorphologie: Candida parapsilosis bildet ovale bis längliche, relativ kleine Blastosporen. Das Pseudomyzel ist meist gut entwickelt. Vereinzelt treten kettenartige Pseudomyzelien auf, die aus überwiegend großen, breiten Zellen bestehen und ein typisches Merkmal für diese Spezies darstellen ( $\bullet$ Abb. 16).

Tab.5 Biochemisches Verhalten von Candida parapsilosis.

\begin{tabular}{|llll} 
Fermentation & & & Assimilation \\
\hline Glukose + & Laktose - & Glukose + & Laktose - \\
\hline Galaktose V & Raffinose - & Galaktose + & Raffinose - \\
\hline Saccharose - & & Saccharose + & Trechalose + \\
\hline Maltose - & & Maltose + & Nitrat -
\end{tabular}

\section{Candida tropicalis}

Candida tropicalis ( $\bullet$ Abb.17-19) wird vor allem in Schleimhautabstrichen und im Sputum nachgewiesen. Oft vermehrt er sich auf den Schleimhäuten in unphysiologischer Weise und kann spezifische mykotische Prozesse verursachen. Auch bei Candida-Mykosen der Haut, insbesondere im Bereich der Interdigitalräume der Hände sowie bei Paronychien ist dieser Sprosspilz gelegentlich beteiligt.

Mikromorphologie: Die Blastosporen sind überwiegend kurzoval und in Gruppen von durchschnittlich 2-5 Zellen im Bereich der Trennwände um das meist gut entwickelte Pseudomyzel angeordnet; Myzel wird ebenfalls gebildet ( $\bullet$ Abb.20).

Tab. 6 Biochemisches Verhalten von Candida tropicalis.

\begin{tabular}{|c|c|c|c|}
\hline \multicolumn{3}{|l|}{ Fermentation } & \multirow{2}{*}{$\begin{array}{l}\text { Assimilation } \\
\text { Laktose - }\end{array}$} \\
\hline Glukose + & Laktose - & Glukose + & \\
\hline $\begin{array}{l}\text { Galaktose + } \\
\text { (schwach) }\end{array}$ & Raffinose - & Galaktose + & Raffinose - \\
\hline Saccharose + & & Saccharose + & Trechalose + \\
\hline Maltose + & & Maltose + & Nitrat - \\
\hline
\end{tabular}

\section{Gattung Cryptococcus \\ $\nabla$}

Zur Gattung Cryptococcus gehören über 30 Arten, wovon Cryptococcus neoformans die humanpathogene Spezies ist [6].

Humanmedizinisch wenig relevante Arten sind u. a. Cryptococcus (C.) albidus, C. laurentii, C. terreus, C. uniguttulatus, C. luteolus und C. gastricus.

Mikroskopisch charakteristisch für diese Pilze ist vor allem das Vorhandensein von Blastosporen, die von einer mukösen Polysaccharidkapsel umgeben sind. Die Kapselbildung, die unterschiedlich stark ausgeprägt sein kann, ist relativ leicht im Negativverfahren mit dem Tusche-Präparat nach Burri nachweisbar.

\section{Cryptococcus neoformans}

Perfektes Stadium: Filobasidiella neoformans, Filobasidiella bacillispora

Synonym: Cryptococcus homonis, Torula neoformans, Torulopsis neoformans, Torula histolytica und viele andere

Cryptococcus neoformans kommt häufig auf Früchten, Pflanzen (Gräsern und Getreidearten) sowie im Erdboden vor. Das hauptsächliche Erregerreservoir dieses weltweit verbreiteten Keimes findet sich jedoch im Verdauungstrakt verschiedener, speziell domestizierter Vogelarten, wie z.B. von Tauben, Hühnern oder Papageien. Im Gegensatz zur ubiquitären Verbreitung dieser Pilzart sind klinisch relevante Infektionen jedoch relativ selten und kommen vor allem bei Patienten mit malignen Erkrankungen des hämatopoetischen Systems vor, z.B. bei Morbus Hodgkin und besonders im Stadium 3 bei AIDS-Patienten.

Die Infektion wird durch Inhalation sporenhaltiger Stäube übertragen und beginnt primär in der Lunge. Zu klinischen Manifestationen führt am häufigsten die Infektion des Zentralnervensystems (ZNS), da die pulmonalen Veränderungen meist latent oder uncharakteristisch (grippe-, bronchitisähnlich) verlaufen [7]. Nach Disseminierung der Erreger auf hämatogenem und lymphogenem Wege werden außer dem ZNS (insbesondere im Spätstadium der Erkrankung) noch eine Reihe anderer Organe, vor allem die Nebennieren, die Nieren, die Milz, die Leber und das Knochenmark befallen.

Mikromorphologie: Die rundlichen, dünnwandigen Blastosporen

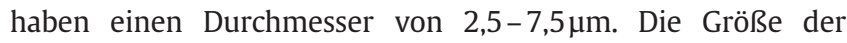
Schleimkapsel kann den Durchmesser der Blastosporen weit übertreffen. Pseudomyzelien und Myzelien werden nicht gebil$\operatorname{det}(\bullet$ Abb.21 und $\odot$ Abb.22).

Cryptococcus neoformans wächst auf Sabouraud-Glukose- (2\%) Agar oder Malzagar bei $20-37^{\circ} \mathrm{C}$ anfangs in weißlich-cremefarbenen Kolonien, die sich allmählich gelbbraun verfärben. $(\bullet$ Abb. 23) Cryptococcus-neoformans-Kolonien verfärben sich auf „Negersaat-Nährböden“ (nach Staib und Seeliger) innerhalb von wenigen Tagen intensiv braun. Die Pigmentbildung, hervorgerufen durch die Inhaltstoffe der Früchte der Komposite Guizotia abyssinica, sowie die Fähigkeit des Wachstums bei $37^{\circ} \mathrm{C}$ stellen besonders wichtige Erkennungsmerkmale zur frühen Identifizierung des Erregers dar.

Tab. 7 Biochemisches Verhalten von Cryptococcus neoformans.

\begin{tabular}{|lll|}
\hline Fermentation & Assimilation \\
\hline fehlt stets & Glukose + & Laktose ${ }^{-}$ \\
\hline Galaktose + & $\begin{array}{l}\text { Raffinose }+ \\
\text { (schwach) }\end{array}$ \\
\hline Saccharose + & Trechalose + \\
\hline Maltose + & Nitrat - \\
\hline
\end{tabular}

\section{Gattung Malassezia (Pityrosporum) \\ Malassezia furfur \\ Synonym: Pityrosporum ovale}

Malassezia furfur, der Erreger der Pityriasis versicolor, einer oberflächlichen, jedoch rezidivfreudigen Dermatomykose ist nach Selliger und Heymer ein weltweit verbreiteter Pilz, der nicht nur in seiner parasitären (Malassezia), sondern auch in der saprophytären Phase (Pityrosporum) beim Menschen auftritt. Die Entwicklung der parasitären Phase bedarf gewisser dispo- 

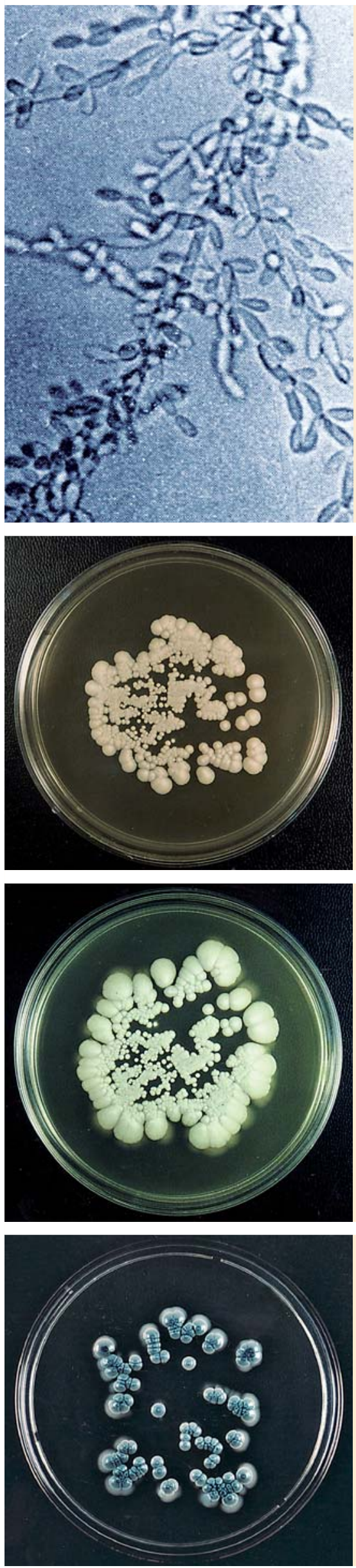

Abb.16 Mikrostrukturen von Candida parapsilosis auf Reisagar (stark vergrößert).

Abb.17 11 Tage alte Reinkultur von Candida tropicalis auf Sabouraud-Glukose-Agar (2\%) (bei $25^{\circ} \mathrm{C}$ ).

Abb.18 20 Tage alte Reinkultur von Candida tropicalis mit randständigen Pseudomyzelausläufern auf SabouraudGlukose-Agar (2\%) (bei $25^{\circ} \mathrm{C}$ ).

Abb.19 Candida tropicalis auf ChromagarCandida (nach 7 Tagen Bebrütung bei $37^{\circ} \mathrm{C}$ ).

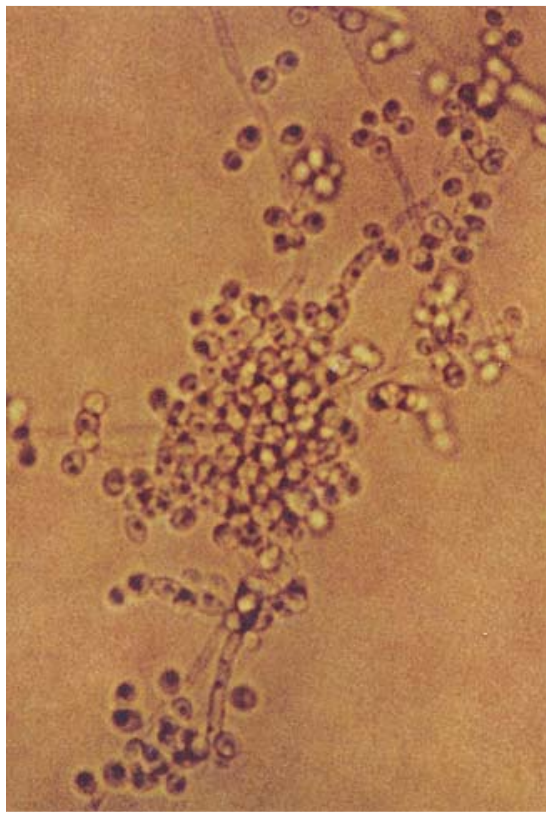

Abb.20 Mikrostrukturen von Candida tropicalis auf Reisagar (stark vergrößert).

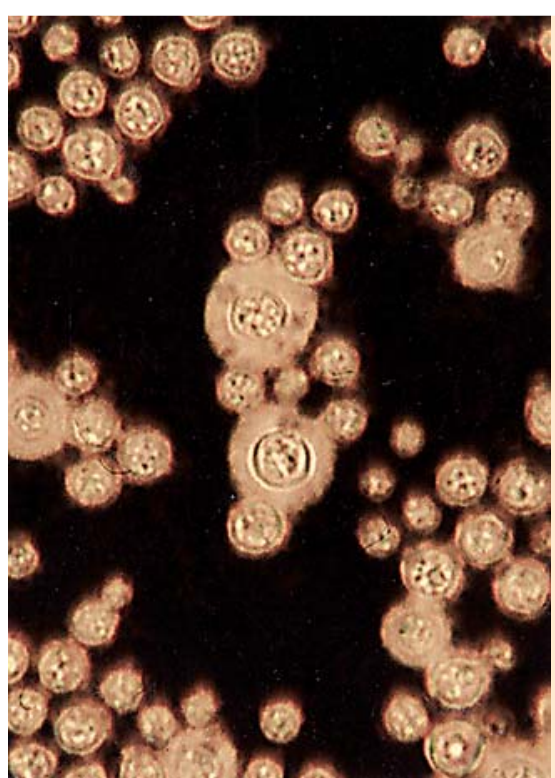

Abb. 21 Tusche-Präparat von Cryptococcus neoformans (stark vergrößert).

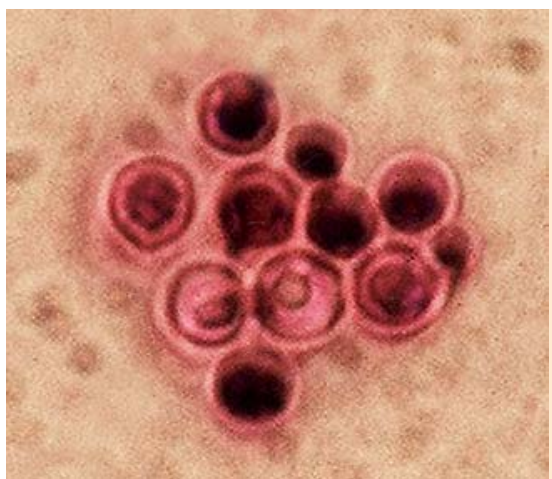

Abb. 22 Methylenblau-Präparat von Cryptococcus neoformans (stark vergrößert). 


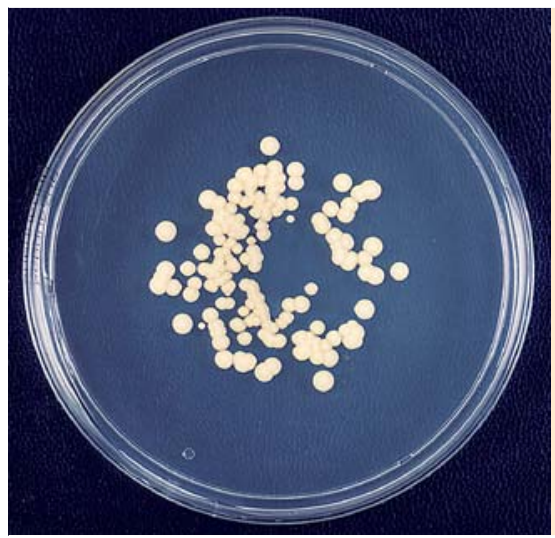

Abb.23 22 Tage alte Reinkultur von Cryptococcus neoformans auf Sabouraud-GlukoseAgar (2\%) (bei $25^{\circ} \mathrm{C}$ ).
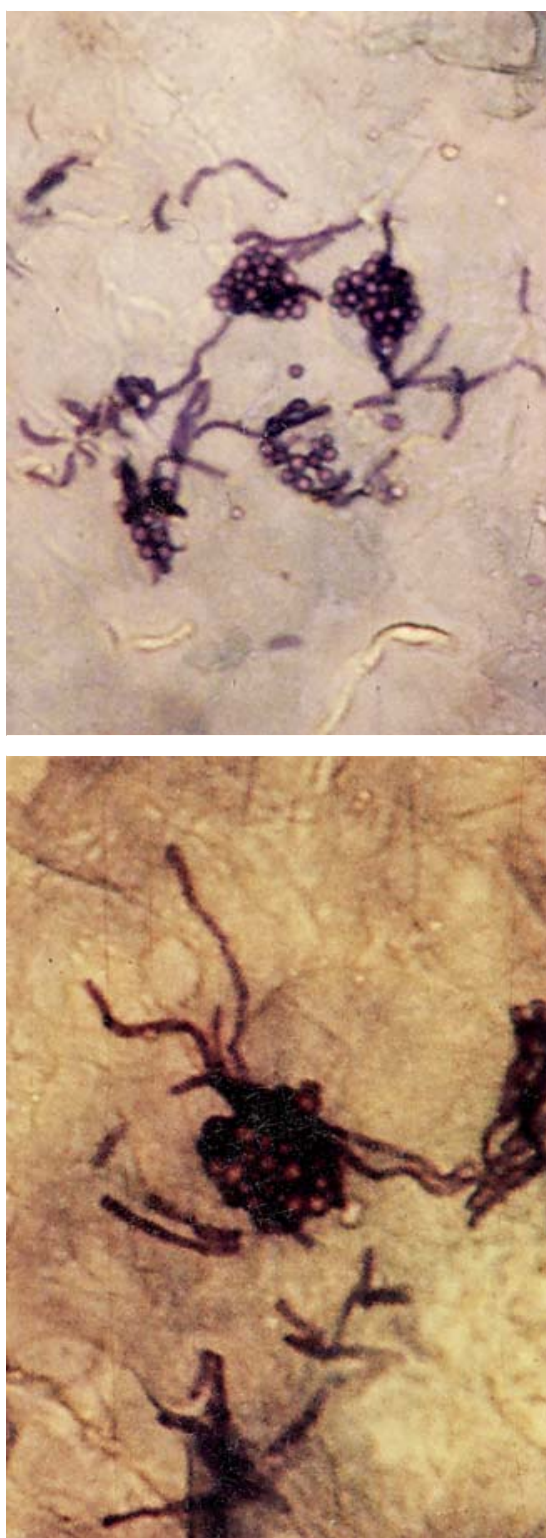

Abb. 25 Hyphen und Sporen von Malassezia furfur (stark vergrößert).

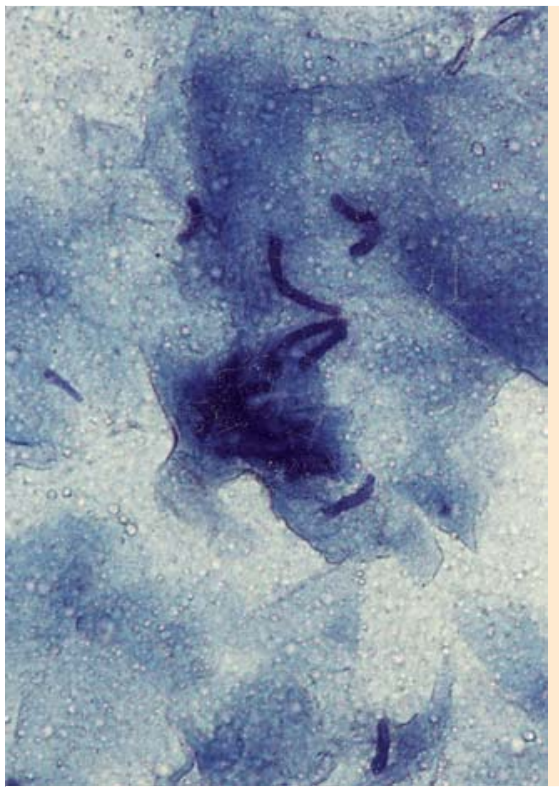

Abb. 26 Halbmondförmig gekrümmte Hyphen von Malassezia furfur.

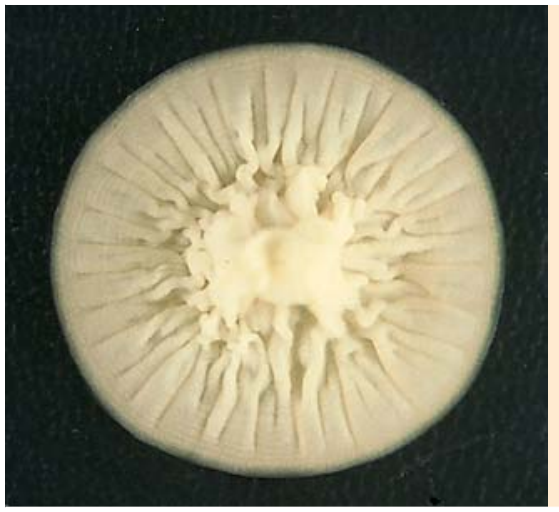

Abb.27 19 Tage alte, weißlich-cremefarbene, stark gefaltete und zerklüftete Monolultur von Trichosporon sp. auf Sabouraud-GlukoseAgar $(2 \%)$ (bei $25^{\circ} \mathrm{C}$ ) (stark vergrößert).

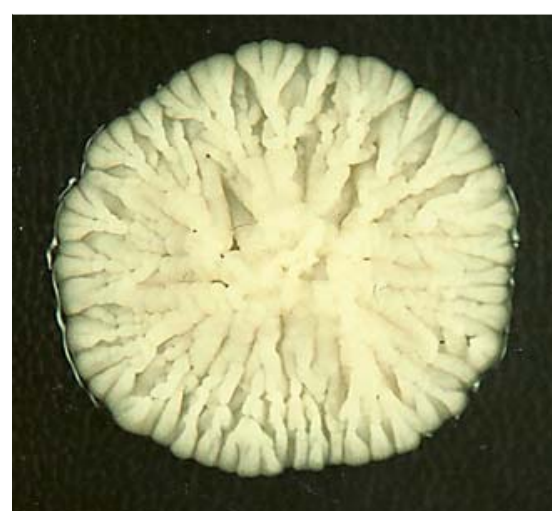

Abb. 2822 Tage alte Monokultur einer anderen TrichosporonArt auf Kimmig-Agar (bei $25^{\circ} \mathrm{C}$ ) (stark vergrößert). 
nierender Faktoren. Hohe $\mathrm{CO}_{2}$-Spannung, das Vorhandensein bestimmter Hautfette sowie die Neigung zum Schwitzen gelten u.a. als begünstigende Bedingungen für das Entstehen dieser Hefepilz-Mykose, die vor allem in tropischen und subtropischen Regionen besonders häufig vorkommt (Befallsquoten bis $60 \%$ ). Bevorzugte Lokalisationen sind die oberen Stammpartien, die Schultern und der Hals. Selten befallen wird dagegen die untere Partie des Stammes.

Direkte mikroskopische Begutachtung: Im Tesafilm-Abrisspräparat findet man als Erscheinungsform von Malassezia furfur runde, doppelt konturierte Sporen von 3-8 $\mu \mathrm{m}$ Durchmesser, die überwiegend nestförmig gelagert sind, sowie relativ kurze, wenig septierte, wurstförmig gekrümmte Myzelfäden ( $\bullet$ Abb.2426).

Die lipidabhängige Hefe wächst auf ölbeschichteten Nährböden in der Hefephase (= Pityrosporum ovale bzw. orbiculare) und bildet innerhalb von wenigen Tagen relativ kleine, weiß-gelbliche, glatte oder raue Kolonien mit unregelmäßigem Randsaum, die aus ellipsoiden und flaschenähnlichen Zellen $(1,5-3 \times 2-5$ bis $3-5 \times 7,7-5,5 \mu \mathrm{m})$ bestehen.

\section{Gattung Trichosporon}

$\nabla$

Diese Gattung umfasst 17 Arten. Trichosporon cutaneum, der Erreger der „weißen Piedra“, stellt die häufigste und medizinisch wichtigste Trichosporon-Spezies dar. Eine pathogene Bedeutung wird auch Trichosporon (T.) asahii, T. asteroides, T. inkin, T. mucoides und T. ovoides zugeschrieben. Diese Sprosspilze treten jedoch nur relativ selten als Krankheitserreger auf. Hefen der Gattung Trichosporon bilden Blastosporen, Pseudomyzel sowie echtes Myzel, das in Gliedersporen (Arthrosporen) zerfällt.

\section{Trichosporon cutaneum}

Bekanntestes Synonym: Trichosporon beigelii

Dieser weit verbreitete Sprosspilz kommt oft in der unmittelbaren Umgebung des Menschen sowie bei tierischen Warmblütlern (Säugetieren und Vögeln) vor.

Im dermatologischen Bereich findet man vor allem Trichosporon cutaneum auf vorgeschädigter oder mazerierter Haut [5]. Trichosporon cutaneum verursacht in Form von weißlich-grauen bis hellbraunen, durchscheinenden, knotigen Auflagerungen eine relativ häufige, jedoch harmlose Infektion der Haarschäfte, die sogenannte Piedra alba ${ }^{1}$. Bei Bestehen disponierender Faktoren, insbesondere starker Schwächung des Immunsystems, z.B. bei AIDS-Patienten, kann dieser Sprosspilz auch gelegentlich Mykosen der Haut und der inneren Organe hervorrufen.

Makromorphologie: Trichosporon cutaneum bildet weiß-gelbliche, stark gefaltete Kolonien mit glänzender oder matter Oberfläche, die schon früh einen strahlenförmigen, in den Agar wachsenden Myzelsaum erkennen lassen ( $\bullet$ Abb.27 und $\bullet$ Abb.28).

Mikromorphologie: Mikroskopisch charakteristisch sind vor allem in Arthrosporen zerfallende Myzelfäden; Blastosporen und Pseudomyzel werden ebenfalls gebildet ( $\bullet$ Abb.29).

\footnotetext{
${ }_{1}^{1}$ Außer Trichosporon cutaneum sind auch Trichosporon inkin und Trichosporon ovoides Erreger der weißen Piedra.
}

Tab. 8 Biochemisches Verhalten von Trichosporon cutaneum.

\begin{tabular}{|lll|}
\hline Fermentation & & Assimilation \\
\hline fehlt stets & Glukose + & Laktose + \\
\hline & Galaktose + & Raffinose + \\
\hline & Saccharose + & Trechalose + \\
\hline & Maltose + & Nitrat - \\
\hline $\begin{array}{l}\text { Harnstoff- } \\
\text { hydrolyse }+\end{array}$ & & \\
\hline
\end{tabular}

\section{Gattung Rhodotorula}

In dieser Gattung unterscheidet man 3 Arten. Charakteristisch für alle Rhodotorula-Spezies ist die Bildung eines karotinoiden Pigments, wodurch die Erkennung in der Kultur, besonders in primären Mischkulturen, erleichtert wird ( $\bullet$ Abb. 30) [8]. Rhodotorula-Arten sind weltweit verbreitet und kommen u.a. in Nahrungsmitteln, in der Luft und im Wasser sowie im Erdboden vor. Im mikroskopischen Kulturpräparat findet man runde, ovale oder längliche Blastosporen ( $\bullet$ Abb.31). Pseudo- und echtes Myzel können zwar gebildet werden, sind aber meist nicht entwickelt [9].

Rhodotorula mucilaginosa ${ }^{2}$, die am häufigsten vorkommende Rhodotorula-Spezies, wurde beim Menschen z. B. in entzündeten Gallenblasen, die frei von Bakterien waren, nachgewiesen. Auch bei Infektionen im Genital-, Intestinal- und Respirationstrakt sowie auf der Haut kann dieser Sprosspilz eine Rolle spielen. Rhodotorula-Spezies sind jedoch als Krankheitserreger zumeist von untergeordneter Bedeutung.

Tab. 9 Biochemisches Verhalten von Rhodotorula mucilaginosa.

\begin{tabular}{|lll|}
\hline Fermentation & & Assimilation \\
\hline fehlt stets & Glukose + & Laktose ${ }^{-}$ \\
\hline & Galaktose V & $\begin{array}{l}\text { Raffinose }+ \\
\text { (schwach) }\end{array}$ \\
\hline & Saccharose + & Trechalose + \\
\hline & Maltose + & Nitrat - \\
\hline
\end{tabular}

\section{Gattung Sporobolomyces}

In dieser Gattung sind 7 Spezies zusammengefasst. Die vegetative Vermehrung erfolgt durch Sprossung sowie durch Schleudersporen, sogenannte Balistosporen, die zur Zeit der Reife etwa $1 \mathrm{~mm}$ weit abgestoßen werden.

Sporobolomyces-Arten sind weit verbreitet und kommen in der Luft, auf Pflanzen sowie in Milch und gezuckerten Getränken vor.

\section{Sporobolomyces salmonicolor}

Perfektes Stadium: Sporidiobolus salmonicolor

Sporobolomyces salmonicolor ist eine rosa pigmentierte, mit Rhodotorula zu verwechselnde Hefe ( $\bullet$ Abb. 32), die gelegentlich auf erkrankter Haut nachgewiesen wird. Dieser Sprosspilz ist jedoch mit großer Wahrscheinlichkeit kein Infektionserreger, sondern lediglich Sekundärbesiedler auf einem bereits vorgeschädigten Terrain.

\footnotetext{
${ }^{2}$ Rhodotorula rubra wurde mit Rhodotorula mucilaginosa zusammengelegt.
} 

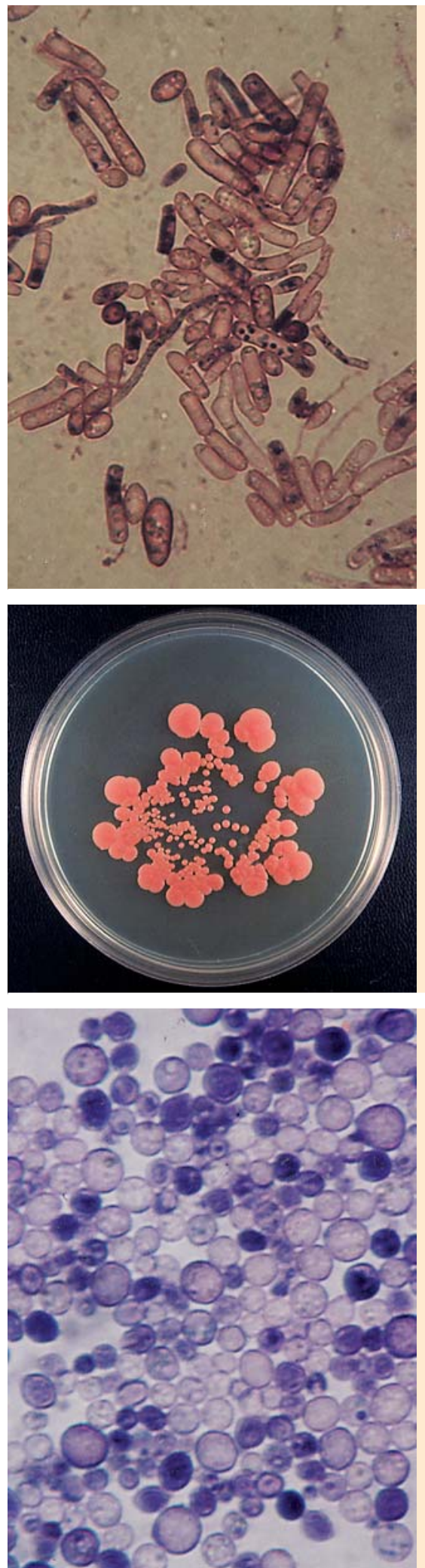

Abb.29 Blastosporen, Arthrosporen und Hyphen von Trichosporon sp. im Methylenblau-Präparat (stark vergrößert).

Abb.30 12 Tage alte Reinkultur von Rhodotorula sp. auf Sabouraud-Glukose-Agar (2\%) (bei $25^{\circ} \mathrm{C}$ ).

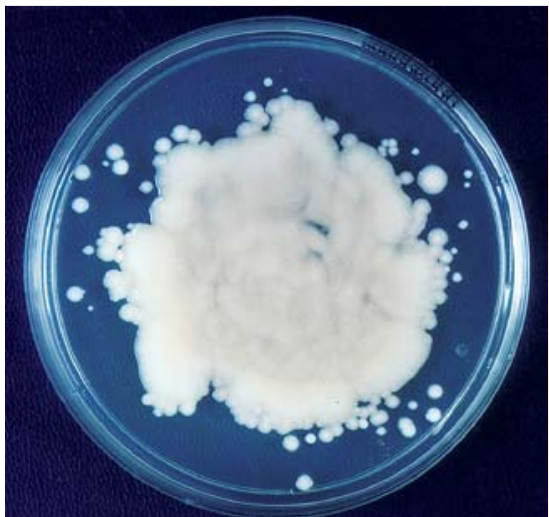

Abb.32 12 Tage alte, schwach rosa pigmentierte Reinkultur von Sporobolomyces salmonicolor auf SabouraudGlukose-Agar (2\%) (bei $25^{\circ} \mathrm{C}$ ).

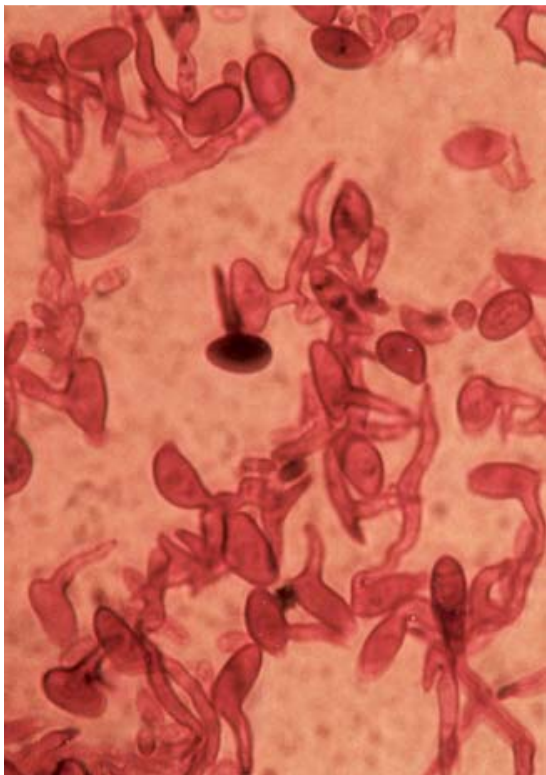

Abb.33 Ballistosporen von Sporobolomyces salmonicolor.

Abb. 31 Blastosporen von Rhodotorula sp. im Methylenblau-Präparat (stark vergrößert). 
Mikromorphologie: Die Blastosporen sind überwiegend langgestreckt und an beiden Polen verjüngt. Mikroskopisch charakteristisch ist vor allem die Entwicklung sichel- oder nierenförmiger Balistosporen, die von der Myzelspitze mithilfe eines Flüssigkeitstropfens abgeschleudert werden ( $\bullet$ Abb.33).

Tab.10 Biochemisches Verhalten von Sporobolomyces salmonicolor.

\begin{tabular}{|c|c|c|}
\hline Fermentation & & Assimilation \\
\hline \multirow[t]{4}{*}{ fehlt stets } & Glukose + & Laktose - \\
\hline & Galaktose V & Raffinose V \\
\hline & Saccharose + & Trechalose + \\
\hline & Maltose V & Nitrat + \\
\hline
\end{tabular}

\section{Abstract}

\section{Introduction to the Diagnosis of Human Pathogenic Fungi - Part 3: Opportunistic Pathogenic Yeasts}

Yeasts are found throughout the world and are common in food, above all in dairy products, but also exist in the air, the water and the soil.

Yeasts that do not form sexual fruiting bodies and only reproduce asexually by means of budding are classified as „fungi imperfecti“ and are referred to as deuteromycota, in contrast to ascomycota, or yeasts that form ascospores.

Imperfect yeasts of the genera Candida, Cryptococcus, Malassezia (Pityrosporum) and, to a certain degree, Rhodotorula and Sporobolomyces are of significance in medicine. The largest genus is Candida, which has some 200 species. Few of these imperfect fungus species are opportunistic parasites and are able to grow at or above $37^{\circ} \mathrm{C}$. These opportunistic pathogens can cause yeasts infections in humans with predisposing risk factors and have become more significant since the introduction of antibiotics, cytostatic drugs, immunosuppressants and other iatrogenic measures.

\section{Literatur}

1 Heber W, Hauss H. Mykologische Techniken in der ärztlichen Praxis. München: Schwarzeck; 1983

2 Koch $H$. Leitfaden der Medizinischen Mykologie. Stuttgart: Fischer; 1981

3 Nolting S, Fegeler K. Medizinische Mykologie. 3. Aufl. Berlin: Springer; 1987

4 Gemeinhardt H (Hrsg). Endomykosen. Stuttgart: Fischer; 1989

5 Seeliger HPR, Heymer T. Diagnostik pathogener Pilze des Menschen und seiner Umwelt. Stuttgart: Thieme; 1981

6 Darai G, Handermann M, Sonntag HG et al. (Hrsg). Lexikon der Infektionskrankheiten des Menschen. Berlin: Springer; 2009

7 Male O. Medizinische Mykologie für die Praxis. Stuttgart: Thieme; 1981

8 Rieth H. Mykologische Diagnostik (Grundkurs/Abschnitt 10). mta-Journal Extra 1984; 5: 37 - 44

9 Kozlowska EA, Nuber D. Leitfaden der praktischen Mykologie. Berlin: Blackwell; 1996 